\section{AML: Nachholbedarf bei der antimykotischen Prophylaxe}

Invasive Pilzinfektionen sind wegen ihrer hohen Morbidität und Mortalität ein großes klinisches Problem bei neutropenischen Patienten mit akuter myeloischer Leukämie (AML) unter intensiver Chemotherapie. Dennoch erhalten selbst Studienpatienten in weniger als zwei Drittel der Fälle die von der European Conference on Infections in Leukemia (ECIL) empfohlene antimykotische Prophylaxe, wie eine prospektive Beobachtungsstudie der Acute Leukemia French Association (ALFA) verdeutlicht [Michallet $M$ et al. ASH. 2016;Abstr 3696].

Ausgewertet wurden Daten von 677 AML-Patienten, die im Rahmen der Studie ALFA 0702 eine intensive InduktionsChemotherapie mit Daunorubicin/Cytarabin erhielten, berichtete Mauricette Michallet, Lyon, Frankreich. Das Studienprotokoll sah eine antimykotische Prophylaxe mit Posaconazol (200 mg 3-mal/ Tag) ab Tag 4 der Induktionstherapie bis zur Neutrophilen-Erholung.

383 der 677 Patienten (57\%) erhielten das Antimykotium über im Schnitt 25 Tage. Knapp ein Drittel der Patienten (30\%) wurde nicht prophylaktisch behandelt. In dieser Gruppe war die kumulative Inzidenz invasiver Aspergillosen
(IA) an Tag 60 mit 8,4\% am höchsten. Bei Patienten, die eine Prophylaxe mit Posaconazol allein oder in Kombination mit einem anderen Antimykotikum erhielten, lag die IA-Rate dagegen nur bei $4,7 \%$. Auch Patienten, die mit einer anderen Substanz als Posaconazol behandelt wurden, waren stärker geschützt als Patienten der ersten Gruppe (IA-Rate $3,3 \%)$.

Nicht unerwartet war das Überleben von Patienten ohne invasive Pilzinfektion nach einem Follow-up von median 27,5 Monaten unabhängig von der Art der Prophylaxe länger als bei denen mit einer infektiösen Episode. Im Falle einer invasiven Pilzinfektion führte die antimykotische Propylaxe zu einer deutlichen Verbesserung der 2-Jahres-Überlebensrate nach Induktionschemotherapie. In der multivariaten Analyse wurden mit ungünstiger Zytogenetik und Auftreten einer invasiven Pilzinfektionen zwei Variablen identifiziert, die die 100-Tage-Sterblichkeit negativ beeinflussten. Katharina Arnheim

Bericht von der 58. Jahrestagung der American Society of Hematology vom 3. bis 6 . Dezember in San Diego, CA/USA enten entwickelten in den nächsten zwölf Monaten ein molekulares Rezidiv, davon neun von 49 Patienten, die nur eine $\mathrm{MR}^{3}$ bei Studieneintritt aufgewiesen hatten, sowie drei von insgesamt 125 Patienten mit zu Beginn stabiler $\mathrm{MR}^{4}$.

Die mediane Zeit bis zum Rezidiv war bei $\mathrm{MR}^{3}$ kürzer als bei $\mathrm{MR}^{4} \mathrm{zu}$ Studienbeginn (median 4,4 vs. 8,7 Monate). Ein Progress $\mathrm{zu}$ einem fortgeschrittenen Krankheitsstadium trat nicht auf, auch wurde kein Verlust des zytogenetischen Ansprechens beobachtet. Alle zwölf $\mathrm{Pa}$ tienten erlangten innerhalb von vier Monaten unter voller TKI-Dosis wieder eine $\mathrm{MR}^{3}$.

Die Halbierung der TKI-Dosis führte in den ersten drei Monaten zu einem deutlichen Rückgang der Nebenwirkungen, die sich danach auf einem niedrigeren Niveau stabilisierten. Nur meist milde muskuloskelettale Nebenwirkungen traten bei Dosisreduktion vorübergehend neu auf - analog eines TKI-Absetzsyndroms. Im nächsten Schritt ist bei
Studienpatienten mit mindestens stabiler $\mathrm{MR}^{3}$ auch unter der halbierten TKIDosis ein Absetzen geplant.

\section{Überleben bei Rituximab- refraktärem FL verlängern}

In der Primäranalyse der offenen PhaseIII-Studie GADOLIN war die Therapie Rituximab-refraktärer indolenter NonHodgkin-Lymphome mit Obinutuzumab plus Bendamustin in der Induktion und anschließender Obinutuzumab-Erhaltungstherapie mit einem Vorteil im PFS gegenüber Bendamustin alleine assoziiert. Die aktualisierte Analyse mit einer um etwa zehn Monate längeren Beobachtungszeit weist jetzt auch auf einen Überlebensvorteil unter der Kombination hin [Cheson B et al. ASH. 2016;Abstr 615]. Von insgesamt 413 randomisierten Patienten waren 335 an einem follikulären Lymphom (FL) erkrankt. Bei dieser Subgruppe war der Median des OS zum jetzigen Auswertungszeitpunkt unter der Kombination noch nicht erreicht, während er im Kontrollarm bei 53,9 Monaten lag (Hazard Ratio [HR] 0,58, $95 \%$-Konfidenzintervall [95\%-KI] 0,39-0,86; $\mathrm{p}=0,0061)$. In der Gesamtkohorte war der Median des OS dagegen in beiden Armen noch nicht erreicht, das PFS mit median 25,8 Monaten weiter signifikant länger als unter Bendamustin alleine (14,1 Monate; HR 0,57, $95 \%$-KI 0,44-0,73; p < 0,0001). In der FL-Subgruppe war der PFS-Unterschied ähnlich (25,3 vs. 14,0 Monate; HR 0,52, $95 \%$-KI 0,39-0,69; $\mathrm{p}<0,0001)$.

\section{JAK-Inhibition bei MF: Spät eingesetzt weniger wirksam}

Patienten mit Myelofibrose (MF), deren Erkrankung weiter fortgeschritten ist, sprechen im klinischen Alltag schlechter auf den Januskinase(JAK)-Inhibitor Ruxolitinib an als Patienten mit noch geringerer Splenomegalie oder Symptomlast. Das belegt eine italienische Beobachtungsstudie mit $408 \mathrm{MF}-P a t i e n t e n] \mathrm{Pa}-$ landri F et al. ASH. 2016;Abstr 1128]. In der multivariaten Analyse korrelierte das Milzansprechen negativ mit folgenden zu Studienbeginn bestimmten Faktoren: _einer ausgeprägten Splenomegalie (Odds Ratio [OR] 0,49, 95 \%-KI 0,27$0,91 ; \mathrm{p}=0,024)$,

_einer Transfusionsabhängigkeit (OR 0,54, $95 \%$-KI 0,32-0,90; $\mathrm{p}=0,017$ ),

- einer Verzögerung des Behandlungsbeginns um $\geq$ zwei Jahre (OR 0,61, 95\%-KI 0,33-0,94; $\mathrm{p}=0,028$ ) und

_einer titrierten Dosis von Ruxolitinib unter $10 \mathrm{mg}$ bid.

Eine negative Assoziation zum Ansprechen der Symptome ergab sich für zwei Faktoren:

_eine Verzögerung des Behandlungsbeginns um $\geq$ zwei Jahre (OR 0,54, $95 \%$-KI 0,29-1,02; $\mathrm{p}=0,056)$ und _zu Therapiebeginn einen hohen Gesamtsymptomwert von über 20 nach dem Instrument MPN-SAF (OR 0,25; 95\%-KI 0,13-0,47; p < 0,001).

Nicht zu lange mit der Therapie warten und auf eine Dosis gemäß der Zulassung auftitrieren ist die Empfehlung von Francesca Palandri, Bologna, Italien.

\section{Friederike Klein}

Bericht von der 58. Jahrestagung der American Society of Hematology vom 3. bis 6. Dezember in San Diego, CA/USA 